\title{
Guillain-Barré syndrome following BNT162b2 COVID-19 vaccine
}

\author{
Michele Trimboli $^{1} \cdot$ Pio Zoleo $^{1} \cdot$ Gennarina Arabia ${ }^{1} \cdot$ Antonio Gambardella $^{1}$ (])
}

Received: 18 April 2021 / Accepted: 25 July 2021 / Published online: 4 August 2021

(c) Fondazione Società Italiana di Neurologia 2021

\section{Dear Editor,}

Guillain-Barré syndrome (GBS) is the most common and severe acute paralytic neuropathy, with about 100,000 people developing the disorder every year worldwide [1]. Up to two-thirds of patients with GBS report an antecedent infection 1-3 weeks prior to the onset of weakness [2]. Six pathogens have been associated with GBS in case-control studies: campylobacter jejuni, cytomegalovirus, hepatitis E virus, mycoplasma pneumoniae, Epstein-Barr, and Zika virus [2-4]. These infections, by either molecular mimicry or bystander activation, are thought to trigger the emblematical GBS immune response resulting in the demyelination of and damage to peripheral nerves [5]. It is possible that other pathogens are linked to GBS, but their role in the pathogenesis of GBS is still uncertain [6, 7].

Vaccines have also been associated to the pathogenesis of GBS as putative triggers [8]. The first epidemiological link between vaccines and GBS was highlighted in 1976 when was reported an increased GBS risk among individuals who received swine flu vaccine [9]. The attributable risk of GBS after influenza vaccination in adults is estimated to be 1-3 in $1,000,000[10,11]$. SARS-CoV-2 is a novel infectious agent causing coronavirus disease 2019 (COVID-19), which has been declared as pandemic in March 2020 [12]. To date, FDA approved three double-dose vaccines for the prevention of COVID-19 infection [BNT162b2 (Pfizer ${ }^{\circledR}$ ); mRNA-1273 (Moderna $\left.{ }^{\circledR}\right)$; Ad26.COV2.S (Johnson \& Johnson $\left.{ }^{\circledR}\right)$ ]. The safety profile of BNT162b2, the first FDA-approved nucleoside-modified messenger RNA vaccine, was characterized by short-term, mild-to-moderate pain at the injection site, fatigue, and headache. The incidence of serious adverse events was low and was similar in the vaccine and placebo groups [13]. However, COVID-19 vaccine-related GBS has

Antonio Gambardella

a.gambardella@unicz.it

1 Department of Medical and Surgical Sciences, Institute of Neurology, Magna Græcia University, V.le Europa, 88100 Catanzaro, Italy been rarely reported to date [14] and some researches disavow the existence of this association [15].

We describe a case of GBS after receiving the second dose of COVID-19 vaccine [BNT162b2-Pfizer $\left.{ }^{\circledR}\right]$. A 25-year-old woman without other comorbidities presented to our department on February 26th 2021 with a history of progressive lower limbs weakness and paresthesias for 4 days. She reported difficulty in walking and climbing stairs associated to paresthesias and numbness in the anterolateral region of the right thigh. The patient had received the first dose of COVID-19 vaccine [BNT162b2-Pfizer®] on January 19th 2021 and the second dose on February 18th 2021. No infectious illness or other events were experienced in the weeks prior to the onset of symptoms. Neurological examination revealed steppage gait due by bilateral feet drop. Motor examination demonstrated normal bulk and tone in bilateral upper and lower extremities, and strength in bilateral upper extremities was noted to be $5 / 5$ on Medical Research Council (MRC) scale in both proximal and distal muscles. Although she was able to sustain her bilateral lower extremities against gravity for over $5 \mathrm{~s}$, the examination of muscle group strength testing showed an important muscle weakness of $2 / 5$ on MRC scale in foot flexors, in particular in tibialis anterior muscle bilaterally. Her sensation to touch and pinprick was intact in bilateral upper and lower extremities but decreased in the anterolateral region of the right thigh. The patient had areflexia in lower extremities while in upper limb had normal reflexes. SARS-CoV-2 RT-PCR was negative and SARS-CoV-2 serology showed negative IgMIgG-IgA antibodies to nucleocapsid protein and positive IgG antibodies to spike protein index: 32.41 (normal value $<1$ ). Complete inflammatory/autoimmune/infective work-up was negative including fecal PCR test for campylobacter jejuni. Brain and spine MRI were both normal. Visual, auditory, motor, and sensory evoked potentials of the upper and lower limbs were unremarkable. Electromyography (EMG) and nerve conduction study were also performed on the day of admission: sensory studies were normal in the upper and lower limbs with the "sural sparing" pattern; motor studies demonstrated conduction block of peroneal nerve bilaterally 
across the fibular head; $\mathrm{F}$ waves in the upper and lower limbs were markedly prolonged in latency, suggesting a demyelinating process affecting the more proximal segments of the nerves, moreover the absence of peroneal nerves $F$ waves confirmed the presence of proximal conduction blocks; needle EMG documented widespread spontaneous activity, subacute neurogenic restructuring of motor unit action potentials, and a reduced interference pattern in tibialis anterior muscle bilaterally. A lumbar puncture was performed and cerebrospinal fluid analysis did not show albuminocytological dissociation. The patient was diagnosed with GBS on the same day of admission and promptly started intravenous immunoglobulin (IVIg) $0.4 \mathrm{~g} / \mathrm{kg} /$ day for 5 days. No complications were observed during and after the treatment and a slight clinical improvement was already appreciated after the first 4 days of intravenous IVIg. The patient received physical therapy during the hospital stay and was thereafter discharged to rehabilitation facility. At the follow-up visit, 30 days later, she reported a substantial clinical improvement. She was again able to climb stairs and difficulty in walking disappeared, and neurological examination showed muscle weakness of $4 / 5$ on MRC scale in tibialis anterior muscle bilaterally. Follow-up NCS demonstrated normalization of $F$ waves latency in the upper and lower limbs, including peroneal nerves $\mathrm{F}$ waves, and left peroneal nerve conduction block disappearance.

As far as we are aware, only one case of GBS has been associated with the COVID vaccines [14]. Thus, it might be questioned if GBS in our patient might represent a mere coincidence following SARS-CoV-2 vaccination. We believe that the clinical and laboratory findings including the lack of overt trigger are consistent with a causal association between GBS and Pfizer ${ }^{\circledR}$ anti-SARS-CoV-2 vaccine [16]. Accordingly, several surveillance studies already illustrated vaccine-related increase in GBS following modern influenza vaccines. Thus, it is possible that additional COVID19 vaccine-associated GBS cases will be described in the near future, even though the individual risk for GBS and other rare complications is likely to be very small, and the benefit of protection against COVID-19 both for individuals and society is far greater. In this way, it might be essential to exploit the opportunity of a worldwide vaccination campaign, perhaps the largest in history, to better understand the pathogenetic mechanism that bind GBS to COVID vaccination, rather than denying such association.

\section{Declarations}

Ethical approval Ethical approval to report this case was obtained from the regional human research ethics committee of the University Magna Græcia, Catanzaro, Italy.
Informed consent Written informed consent was obtained from the patient for her anonymized information to be published in this article.

Conflict of interest The authors declare no competing interests.

\section{References}

1. Willison HJ, Jacobs BC, van Doorn PA (2016) Guillain-Barre syndrome. Lancet 388:717-727

2. Jacobs BC, Rothbarth PH, van der Meché FG et al (1998) The spectrum of antecedent infections in Guillain-Barré syndrome: a case-control study. Neurology 51:1110-1115

3. Cao-Lormeau VM, Blake A, Monset S et al (2016) Guillain-Barré syndrome outbreak associated with Zika virus infection in French Polynesia: a case-control study. Lancet 387:1531-1539

4. Van den Berg B, Van Der Eijk AA, Pas SD et al (2014) GuillainBarrè syndrome associated with preceding hepatitis $E$ virus infection. Neurology 82:491-497

5. Yuki N, Hartung HP (2012) Guillain-Barrè syndrome. N Engl J Med 366:2294-2304

6. Vellozzi C, Iqbal S, Broder K (2014) Guillain-Barré syndrome, influenza, and influenza vaccination: the epidemiologic evidence. Clin Infect Dis 58:1149-1155

7. Cornblath DR, McArthur JC, Kennedy PG, Witte AS, Griffin JW (1987) Inflammatory demyelinating peripheral neuropathies associated with human T-cell lymphotropic virus type III infection. Ann Neurol 21:32-40

8. McGonigal R, Rowan EG, Greenshields KN et al (2010) AntiGD1a antibodies activate complement and calpain to injure distal motor nodes of Ranvier in mice. Brain 133:1944-1960

9. Schonberger LB, Bregman DJ, Sullivan-Bolyai JZ et al (1979) Guillain-Barré syndrome following vaccination in the National Influenza Immunization Program, United States, 1976-1977. Am J Epidemiol 110:105-123

10. Salmon DA, Proschan M, Forshee R et al (2013) Association between Guillain Barrè syndrome and influenza A (H1N1) 2009 monovalent inactivated vaccines in the USA: a metaanalysis. Lancet 381:1461-1468

11. Martín Arias LH, Sanz R, Sáinz M et al (2015) Guillain Barrè syndrome and influenza vaccines: a metaanalysis. Vaccine 33:3773-3778

12. Zhu N, Zhang D, Wang W et al (2019) China Novel Coronavirus Investigation and Research Team (2020) A novel coronavirus from patients with pneumonia in China, 2019. N Engl J Med 382:727-733

13. Polack FP, Thomas SJ, Kitchin N et al (2020) Safety and efficacy of the BNT162b2 mRNA Covid-19 vaccine. N Engl J Med 31:2603-2615

14. Waheed S, Bayas A, Hindi F et al (2021) Neurological complications of COVID-19: Guillain-Barre syndrome following Pfizer COVID-19 vaccine. Cureus 13:e13426

15. Lunn MP, Cornblath DR, Jacobs BC et al (2021) Covid 19 vaccine and Guillan Barrè syndrome: let's not leap association. Brain 144:357-360

16. Chen RT, Rastogi SC, Mullen JR et al (1994) The vaccine adverse event reporting system (VAERS). Vaccine 12:542-550

Publisher's note Springer Nature remains neutral with regard to jurisdictional claims in published maps and institutional affiliations. 